\begin{abstract}
Classification
Physics Abstracts 4.440

\section{PROPERTIES OF THE LOW-LYING NEGATIVE PARITY STATES IN ${ }^{45} \mathrm{Sc}$}

\author{
J. CHEVALliER, B. HAAS, N. SCHULZ and M. TOULEMONDE \\ Centre de Recherches Nucléaires et Université Louis-Pasteur, 67037 Strasbourg, France
}

(Reçu le 24 octobre 1975, accepté le 4 décembre 1975)

\begin{abstract}
Résumé. - Les décroissances des états de parité négative d'énergie d'excitation inférieure à $2107 \mathrm{keV}$ dans le noyau ${ }^{45} \mathrm{Sc}$ ont été étudiées par la réaction ${ }^{42} \mathrm{Ca}(\alpha, \mathrm{p} \gamma){ }^{45} \mathrm{Sc}$ à une énergie de bombardement de $10,5 \mathrm{MeV}$. Les protons ont été détectés dans un compteur annulaire de silicium, à un angle moyen de $171^{\circ}$ par rapport à l'axe de faisceau. Des spectres de rayonnements $\gamma$ ont été mesurés aux angles $105^{\circ} \geqslant \theta \geqslant 0^{\circ}$ au moyen d'un détecteur $\mathrm{Ge}(\mathrm{Li})$. Les spins et les vies moyennes des niveaux ainsi que les rapports d'embranchement et les mélanges multipolaires des transitions $\gamma$ ont été déduits des mesures de corrélations angulaires proton- $\gamma$. Les éléments de matrice réduits des transitions électromagnétiques ont été obtenus pour un certain nombre de transitions. Des calculs basés sur le modèle à couplage fort ont été effectués. Ces résultats sont comparés à l'expérience.
\end{abstract}

\begin{abstract}
The electromagnetic decays of the negative parity states in ${ }^{45} \mathrm{Sc}$ up to an excitation energy of $2107 \mathrm{keV}$ have been investigated via the ${ }^{42} \mathrm{Ca}(\alpha, \mathrm{p} \gamma)^{45} \mathrm{Sc}$ reaction at a bombarding energy of $10.5 \mathrm{MeV}$. Reaction produced protons were detected in an annular silicon detector at an average angle of $171^{\circ}$. Gamma-ray spectra were measured with a $\mathrm{Ge}(\mathrm{Li})$ detector at angles $105^{\circ} \geqslant \theta \geqslant 0^{\circ}$. Spins and lifetimes of the levels as well as branching and mixing ratios of their decay $\gamma$-rays have been obtained from proton-gamma angular correlation measurements. Reduced electromagnetic transition matrix elements have been extracted for a number of observed transitions. Calculations based on the strong coupling model have been performed. The results are compared with experiment.
\end{abstract}

1. Introduction. - The low-lying negative parity states in the ${ }^{45} \mathrm{Sc}$ nucleus have been the subject of several theoretical investigations. Both the spherical shell model of McCullen, Bayman and Zamick [1] who considered 5 particles coupled in the $1 \mathrm{f}_{7 / 2}$ shell, and the strong-coupling deformed rotator model of Malik and Scholz [2] have been used in order to explain the negative parity level scheme. However insufficient experimental data were available to allow a very detailed test of these models.

The energy levels and their decay properties have been investigated by many authors. When this work was begun the information available on the ${ }^{45} \mathrm{Sc}$ nucleus had been obtained from the following experiments : Coulomb excitation [3-10], beta-decay [9], $[11,12]$, radiative proton capture [13-16], inelastic proton scattering [10], [17-21], one or two particle transfer reactions [22-25] and the $(\alpha, \mathrm{p} \gamma)$ reaction $[26,27]$. The low-lying negative parity states from $I^{\pi}=1 / 2^{-}$to $15 / 2^{-}$have been extensively discussed in all these papers. However, many ambiguities concerning spin assignments existed and the electromagnetic properties had scarcely been investigated. During the course of the present work,
Metzger [28], using the nuclear resonance fluorescence technique, deduced lifetimes for some excited states in disagreement with previous values measured by the Doppler shift attenuation method. Finally, some high-spin states have recently been populated in heavy-ion induced fusion evaporation reactions [29].

In order to get more information on the negative parity states up to $2107 \mathrm{keV}$, lifetimes, decay modes and spin values were measured using the ${ }^{42} \mathrm{Ca}(\alpha, \mathrm{p} \gamma)$ ${ }^{45} \mathrm{Sc}$ reaction. The experimental procedure and data analysis will be presented in section 2 . The results of the present work are given in section 3 . In the last section, the level scheme and the electromagnetic properties will be compared to the results of a new calculation in the framework of the strong coupling model.

Positive parity states were also populated in this reaction; their properties will be presented and discussed in a separate paper (M. Toulemonde et al., to be published).

2. Experimental procedure and data analysis. 2.1 EXPERIMENTAL PROCEDURE. - The excited states of ${ }^{45} \mathrm{Sc}$ were populated by the ${ }^{42} \mathrm{Ca}(\alpha, \mathrm{p} \gamma)^{45} \mathrm{Sc}$ reac- 
tion at a bombarding energy of $10.5 \mathrm{MeV}$. The doubly ionized ${ }^{4} \mathrm{He}$ particle beam, provided by the $5.5 \mathrm{MV}$ Van de Graaf accelerator of Strasbourg, had an intensity of about $150 \mathrm{nA}$. The target, positioned at $45^{\circ}$ to the beam axis, consisted of a $150 \mu \mathrm{g} / \mathrm{cm}^{2}$ metallic calcium film (enriched to $>92 \%$ in ${ }^{42} \mathrm{Ca}$ ) evaporated on a $500 \mu \mathrm{g} / \mathrm{cm}^{2}$ metallic $\mathrm{Ba}$ backing in order to stop the recoiling ${ }^{45} \mathrm{Sc}$ ions. This backing was deposited on a thick tantalum foil which stopped the beam. To prevent the target from oxidation, it was covered with a $100 \mu \mathrm{g} / \mathrm{cm}^{2}$ gold layer.

Protons from the reaction were detected in a $150 \mathrm{~mm}^{2}$ annular silicon counter placed at $180^{\circ}$ with respect to the beam direction and $3.5 \mathrm{~cm}$ from the target. To eliminate the backscattered $\alpha$-particles, the detector was covered with an $25 \mathrm{mg} / \mathrm{cm}^{2} \mathrm{Al}$ foil. Under these conditions, the energy resolution of the particle detection system was approximately $170 \mathrm{keV}$.

A $80 \mathrm{~cm}^{3} \mathrm{Ge}(\mathrm{Li})$ counter, mounted on a movable table at $6.5 \mathrm{~cm}$ from the target center, was used to measure the $\gamma$-ray yield at $\theta=0^{\circ}, 35^{\circ}, 55^{\circ}, 90^{\circ}$ and $105^{\circ}$ to the beam direction. The energy resolution of this detector was $3.5 \mathrm{keV}$ for the $1.33 \mathrm{MeV}{ }^{60} \mathrm{Co}$ $\gamma$-rays. The instrumental anisotropy of the arrangement was determined by measuring the angular correlation of the $\gamma$-transitions from the $I^{\pi}=1 / 2^{+}$, $934.9 \mathrm{keV}$ level in ${ }^{45} \mathrm{Sc}$.

The proton- $\gamma$ coincidences were collected in a dual parameter system as described elsewhere [30]. The $\gamma$-ray spectra were stored in 4096 channels and the proton spectra in 118. As an example, the spectrum of $\gamma$-rays, at $\theta=90^{\circ}$, in coincidence with protons feeding the 377 and $543 \mathrm{keV}$ levels is presented in figure 1 . It should be pointed out that the difficulty in the analysis of the angular correlation measurements of Zuk et al. [26] is removed in the present case. Because of the small energy difference of the $I^{\pi}=7 / 2^{-}$, ground state and the $I^{\pi}=3 / 2^{+}$, first excited state $(12.4 \mathrm{keV})$, the transitions desexciting a level to both

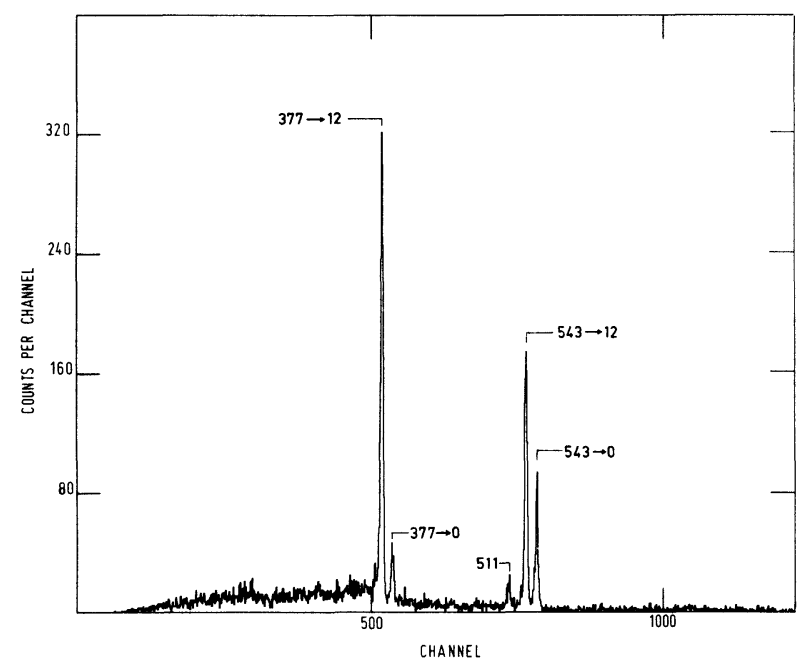

FIG. 1. - Coincidence $\mathrm{Ge}(\mathrm{Li})$ spectrum at $\theta=90^{\circ}$ resulting from the decay of the 377 and $543 \mathrm{keV}$ levels in ${ }^{45} \mathrm{Sc}$. these states could not be resolved in the $\mathrm{NaI}$ detector. As can be seen in figure 1, such transitions are easily analysed in the present experiment.

2.2 ANGUlar CORRELATION ANALYSIS. - Since the outgoing protons were detected at $\left\langle\theta_{\mathrm{p}}\right\rangle=171^{\circ}$, magnetic substates $m= \pm 1 / 2$ were populated. Following the arguments given by Ball et al. [31] for a similar study of the ${ }^{40} \mathrm{Ca}(\alpha, \mathrm{p} \gamma){ }^{43} \mathrm{Sc}$ reaction, the contribution from the $m= \pm 3 / 2$ substrates, due to the finite size of the particle detector, can be neglected in this experiment. The experimental angular correlations for the strongest transitions in ${ }^{45} \mathrm{Sc}$ (an example is shown in the upper part of figure 2) were fitted to a series of Legendre polynomials. The coefficients of these polynomials are listed in table I while the branching ratios extracted from them are given in table II. For the very weak transitions, these values (or limits) were derived from the $\theta=55^{\circ}$ measurement. The allowed values of $I$ and $\delta$ are based on a $\chi^{2}$ analysis of the angular correlations [32]. An example of the $\chi^{2}$ plot for two spin sequences for the $720.6 \mathrm{keV}$ transition is shown in the lower part of
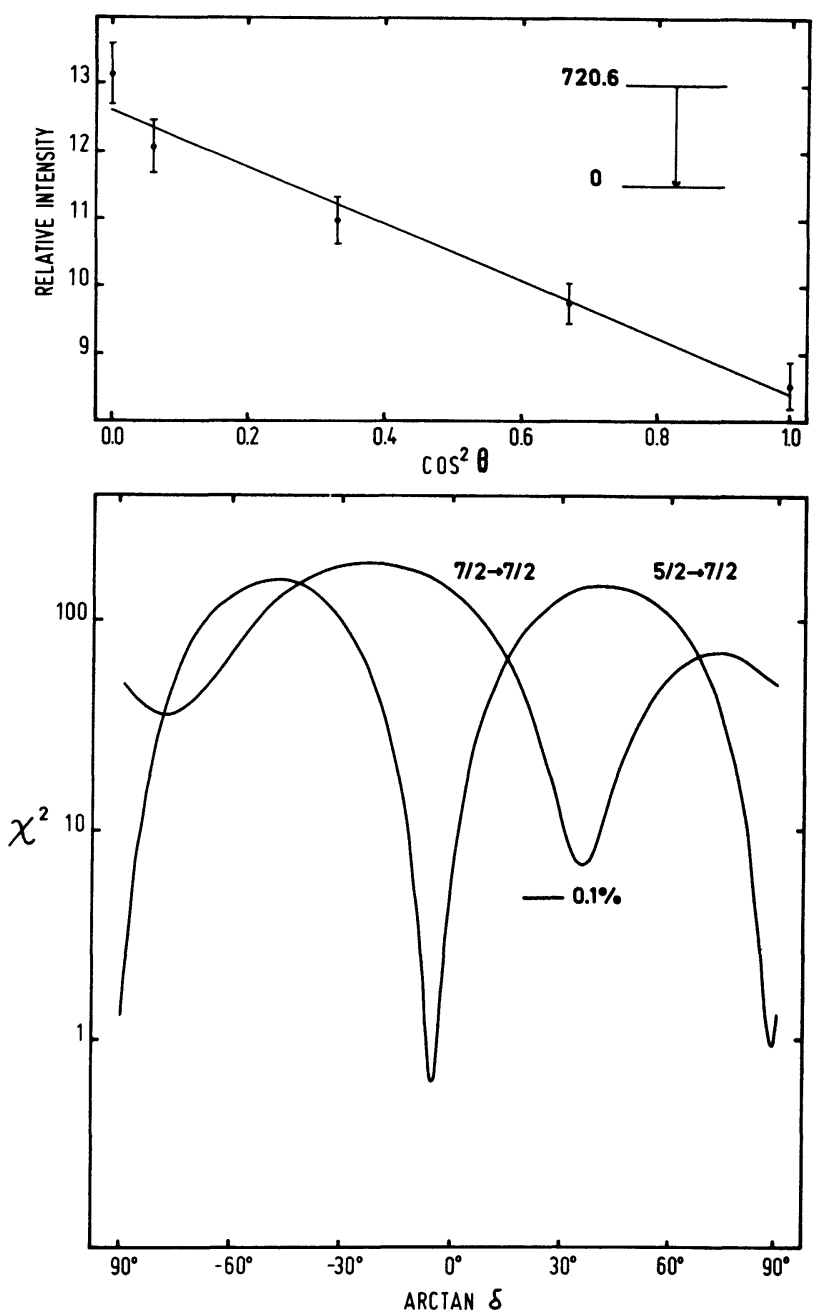

Fig. 2. - Measured angular correlation for the $720.6 \mathrm{keV} \gamma$ transition, along with the Legendre polynomial fit (upper part) and $\chi^{2}$ plot for different spin sequences (lower part). 
TABLE I

Results of least squares Legendre polynomial fits to the proton- $\gamma$ angular correlations

\begin{tabular}{crrr}
$E_{\mathrm{i}}(\mathrm{keV})$ & $E_{\mathrm{f}}(\mathrm{keV})$ & \multicolumn{1}{c}{$A_{2} / A_{\mathbf{0}}\left({ }^{a}\right)$} & $A_{4} / A_{0}\left({ }^{a}\right)$ \\
- & - & $0.36 \pm 0.03$ & - \\
377 & 0 & $0.24 \pm 0.11$ & \\
& 12 & $-0.30 \pm 0.03$ & \\
721 & 0 & $-0.17 \pm 0.28$ & \\
& 12 & $0.20 \pm 0.04$ & \\
1069 & 377 & $-0.13 \pm 0.07$ & \\
& 721 & $0.45 \pm 0.04$ & $-0.18 \pm 0.04$ \\
1237 & 0 & $0.47 \pm 0.04$ & \\
1409 & 0 & $-0.98 \pm 0.06$ & \\
1557 & 1069 & 0.04 & $0.09 \pm 0.03$ \\
1662 & 12 & $-0.14 \pm 0.06$ & \\
& & $0.05 \pm 0.04$ & \\
2092 & 0 & -0.05 & \\
& 1069 & $-0.37 \pm 0.18$ & \\
2107 & 1237 & $0.40 \pm 0.05$ & $-0.22 \pm 0.06$
\end{tabular}

( $\left.{ }^{a}\right)$ These values have not been corrected for the finite solid angle attenuation factors $Q_{2}=0.92$ and $Q_{4}=0.78$ for the $\mathrm{Ge}(\mathrm{Li})$ detector.

figure 2. Spin values were discarded if the corresponding $\chi_{\min }^{2}$ was not within the $0.1 \%$ confidence limit. Signs of $\delta$ are given according to the phase convention of Rose and Brink [33]. The errors associated with $\delta$ were obtained at a $\chi^{2}$ value corresponding to one standard deviation from $\chi_{\min }^{2}$. In addition possible spin sequences and mixing ratios were rejected on the basis of unacceptable transition strengths [34].

2.3 LifETIME anAlysis. - As a check on possible gain shifts in the $\mathrm{Ge}(\mathrm{Li})$ system and in order to get the energy calibration, ${ }^{56} \mathrm{Co} \gamma$-rays coincident in the $\mathrm{Ge}(\mathrm{Li})$ counter and in a $5 \times 5 \mathrm{~cm} \mathrm{NaI}(\mathrm{Tl})$ crystal, shielded from the beam, were simultaneously recorded. Peak positions measured at the different angles were determined from first moment calculations. Each coincident $\gamma$ spectrum was calibrated and calibration errors were quadratically added to the errors found in the center of gravity determination. The full Doppler shifts were computed from the kinematics. The average recoil velocity of the ${ }^{45} \mathrm{Sc}$ ions was $\langle\beta\rangle=\langle v / c\rangle \simeq 0.9 \%$. The experimental attenuation factors $F(\tau)$ and the unshifted $\gamma$-ray energies were computed from least-squares fits to the experimental points versus $\cos \theta$ (Fig. 3). The resulting values are given in table II.

The attenuation factor $F(\tau)$ was computed as a

TABLE II

Excitation energies, branching ratios and lifetimes

\begin{tabular}{|c|c|c|c|c|c|c|c|c|}
\hline $\begin{array}{c}\text { Initial } \\
\text { level } \\
\text { (keV) }\end{array}$ & $\begin{array}{l}\text { Final } \\
\text { level } \\
(\mathrm{keV})\end{array}$ & $\begin{array}{c}\text { Branching ratios } \\
(\%) \\
\text { this work }\end{array}$ & $\begin{array}{c}F(\tau) \\
(\%) \\
\text { this work }\end{array}$ & $\begin{array}{c}(\alpha, p \gamma) \\
\text { this work }\end{array}$ & $\begin{array}{l}\left(p, p^{\prime} \gamma\right) \\
\text { ref. }[21]\end{array}$ & $\begin{array}{c}\quad \tau(\mathrm{fs}) \\
(\mathrm{p}, \gamma) \\
\text { ref. [15] }\end{array}$ & $\begin{array}{c}(\gamma, \gamma) \\
\text { ref. [28] }\end{array}$ & $\begin{array}{l}\text { (C.E.) } \\
\left({ }^{a}\right)\end{array}$ \\
\hline $376 . \overline{8} \pm 0.3$ & $\begin{array}{l}\overline{0} \\
12.4\end{array}$ & $\begin{array}{r}8.6 \pm 0.5 \\
91.4 \pm 0.5\end{array}$ & $\leqslant 9$ & $>\overline{6000}$ & $>\overline{1600}$ & - & - & $65000 \pm 5000$ \\
\hline $720.6 \pm 0.5$ & $\begin{array}{l}0 \\
12.4 \\
\text { other }\end{array}$ & $\begin{array}{l}96.5 \pm 0.5 \\
3.5 \pm 0.5 \\
<2\end{array}$ & $\begin{array}{l}75 \pm 2 \\
86 \pm 9\end{array}$ & $360_{-80}^{+110}$ & $220 \pm 60$ & & $320 \pm 30$ & \\
\hline $1068.6 \pm 1.0$ & $\begin{array}{r}376.8 \\
720.6 \\
\text { other }\end{array}$ & $\begin{array}{c}76 \pm 2 \\
24 \pm 2 \\
<10\end{array}$ & $\begin{array}{l}66 \pm 3 \\
66 \pm 10\end{array}$ & $500_{-120}^{+180}$ & & & & \\
\hline $1237.5 \pm 0.5$ & $\begin{array}{c}0 \\
\text { other }\end{array}$ & $\begin{array}{l}100 \\
<10\end{array}$ & $18 \pm 2$ & $3400_{-900}^{+1400}$ & $1100 \pm 200$ & & $2630 \pm 200$ & $3100 \pm 600$ \\
\hline $1409.5 \pm 0.5$ & $\begin{array}{c}0 \\
720.6 \\
\text { other }\end{array}$ & $\begin{array}{c}87 \pm 4 \\
13 \pm 4 \\
<10\end{array}$ & $60 \pm 2$ & $620_{-120}^{+180}$ & $600 \pm 300$ & $\leqslant 172$ & $400 \pm 30$ & \\
\hline $1557.1 \pm 1.0$ & $\begin{array}{c}1068.6 \\
\text { other }\left({ }^{b}\right)\end{array}$ & $\begin{array}{l}100 \\
<8\end{array}$ & $72 \pm 4$ & $400+180$ & & & & \\
\hline $1662.0 \pm 0.5$ & $\begin{array}{c}0 \\
1237.5 \\
\text { other }\end{array}$ & $\begin{array}{c}79 \pm 3 \\
21 \pm 3 \\
<5\end{array}$ & $85 \pm 2$ & $200 \pm 70$ & $110 \pm 30$ & & $151 \pm 13$ & \\
\hline $2092.3 \pm 1.0$ & $\begin{array}{c}0 \\
376.8 \\
1068.6 \\
\text { other }\left({ }^{c}\right)\end{array}$ & $\begin{aligned} & 83 \pm 2 \\
& 7 \pm 2 \\
& 10 \pm 2 \\
&<10\end{aligned}$ & $\begin{array}{l}100 \pm 1 \\
105 \pm 7\end{array}$ & $<50$ & & $12 \pm 3$ & $8 \pm 1$ & \\
\hline $2106.8 \pm 0.6$ & $\begin{array}{l}1237.5 \\
\text { other }\end{array}$ & $\begin{array}{l}100 \\
<15\end{array}$ & $2 \pm 2$ & $>8000$ & & & & \\
\hline
\end{tabular}

$\left({ }^{a}\right)$ The lifetime values have been calculated using the weighted average of the $B(\mathrm{E} 2)$ values obtained Coulomb excitation experiments [3-10]; only the pure E2- $\gamma$ transitions have been considered.

(b) The branching ratio for the transition to the $543.3 \mathrm{keV}\left(5 / 2^{+}\right)$level is less than $20 \%$.

(c) The branching ratio for the transition to the $1303.5 \mathrm{keV}\left(3 / 2^{+}, 5 / 2^{+}\right)$level is less than $25 \%$. 


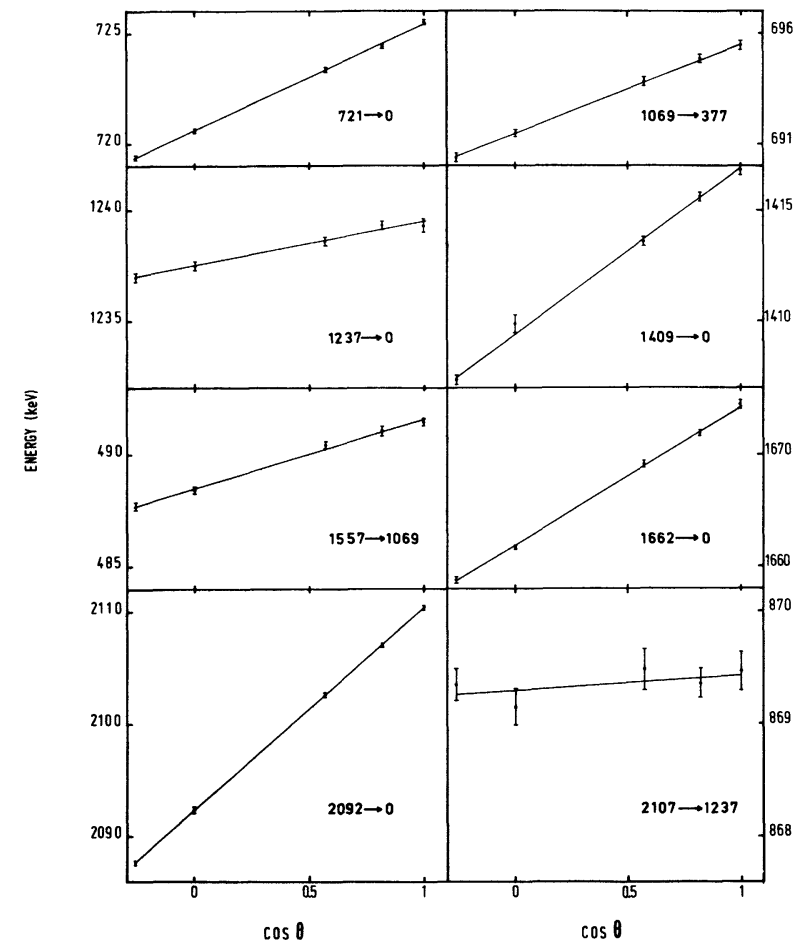

FIG. 3. - Attenuated Doppler shifts for some $\gamma$-transitions desexciting negative parity states. The solid lines are least squares fits to the measured energies.

function of $\tau$ with the stopping theory of Lindhard, Scharff and Schiott [35] including the Blaugrund approximation [36]. Recently Broude et al. [37] and Haas et al. [38] have shown that lifetime values deduced from the Doppler-shift attenuation method (DSAM) can vary depending on the slowing-down environment. However the values obtained with calcium or barium as the slowing down material agree with those obtained in an independent way, namely the recoil distance method (RDM). ${ }^{40} \mathrm{Ca}$ being a contaminant of the present target, the attenuated Doppler shifts for the $\gamma$-rays desexciting the $1159 \mathrm{keV}$ level in ${ }^{43} \mathrm{Sc}$, populated via the ${ }^{40} \mathrm{Ca}(\alpha, \mathrm{p} \gamma)$ reaction, were analysed. An experimental attenuation factor $F(\tau)=0.12 \pm 0.04$ leads to the following lifetime $\tau=5.4_{-1.2}^{+2.8} \mathrm{ps}$ without any correction to the stopping power. A value $\tau=6.4 \pm 1.5 \mathrm{ps}$ has been determined previously by RDM [39]. The agreement indicates a correct phenomenological treatment of the stopping power for ${ }^{45} \mathrm{Sc}$ ions recoiling in calcium and barium. Therefore, no correction factors $f_{\mathrm{n}}$ and $f_{\mathrm{e}}$ were applied to the nucleon and electronic stopping powers. Limits assigned to the mean lives were obtained assuming $20 \%$ uncertainty in the stopping powers. The present lifetime values as well as previous results are presented in table II.

3. Results. - A summary of the experimental results obtained in the present work and a comparison with previous data are given in tables II and III. The over-all agreement between the present lifetime

\section{TABLE III}

Spin and multipole mixing ratio values derived from the present angular correlation measurements. Lifetime and other arguments limiting $I^{\pi}$ and $\delta$ values are included (see text).

\begin{tabular}{crrcc}
$E_{\mathrm{i}}(\mathrm{keV})$ & $E_{\mathrm{f}}(\mathrm{keV})$ & \multicolumn{1}{c}{$I_{\mathrm{i}}^{\pi}$} & $I_{\mathrm{f}}^{\pi}$ & $\delta$ \\
- & - & \multicolumn{1}{c}{-} & - & - \\
377 & 0 & $3 / 2^{-}$ & $7 / 2^{-}$ & $-0.01 \pm 0.08$ \\
& 12 & $3 / 2^{-}$ & $3 / 2^{+}$ & $0.01 \pm 0.02$ \\
721 & 0 & $5 / 2^{-}$ & $7 / 2^{-}$ & $-0.088 \pm 0.055$ \\
1069 & 377 & $3 / 2^{-}$ & $3 / 2^{-}$ & $0.11 \pm 0.05$ \\
& 721 & $3 / 2^{-}$ & $5 / 2^{-}$ & $-0.04 \pm 0.13$ \\
1237 & 0 & $11 / 2^{-}$ & $7 / 2^{-}$ & $0.02 \pm 0.02$ \\
1409 & 0 & $5 / 2^{-}$ & $7 / 2^{-}$ & $0.9 \pm 0.4$ \\
& & $7 / 2^{-}$ & $7 / 2^{-}$ & $-0.05_{-0.13}^{+0.09}$ \\
1557 & 1069 & $1 / 2^{-}$ & $3 / 2^{-}$ & unconstrained \\
1662 & 0 & $9 / 2^{-}$ & $7 / 2^{-}$ & $0.47 \pm 0.05$ \\
& 1237 & $9 / 2^{-}$ & $11 / 2^{-}$ & $0.03 \pm 0.13$ \\
2092 & 0 & $5 / 2^{-}$ & $7 / 2^{-}$ & $0.05 \pm 0.06$ \\
2107 & 1069 & $5 / 2^{-}$ & $3 / 2^{-}$ & $0.05 \pm 0.20$ \\
& 1237 & $15 / 2^{-}$ & $11 / 2^{-}$ & $0.02 \pm 0.05$
\end{tabular}

values and those obtained in the resonance fluorescence experiments is good. It should be pointed out that our reported lifetime values for the 1237 and $1409 \mathrm{keV}$ levels are in disagreement with the values determined via the $\left(p, p^{\prime} \gamma\right)$ and $(p, \gamma)$ reactions respectively. This difference may come from the treatment of the stopping power in the case of recoiling ions with lower velocities $(\langle\beta\rangle \simeq 0.4 \%$ for the first reaction, $\langle\beta\rangle \simeq 0.1 \%$ for the second one). Furthermore since the $1237 \mathrm{keV}$ transition is pure E2, additional lifetime information is directly available from Coulomb excitation experiments [310]; the weighted average of the reported reduced E2 transition probabilities, B(E2) $\uparrow=138 \pm 28 \mathrm{e}^{2} \mathrm{fm}^{4}$, corresponds to a lifetime $\tau=3100 \pm 600 \mathrm{fs}$, in agreement with the present determined value. As pointed out earlier, calcium and barium seem to be slowing down materials providing correct lifetime values using the Doppler shift attenuation method; this leads to a greater confidence in the experimental transition probabilities determined in the present work.

Another general remark concerns the dipole to quadrupole mixing ratios $\delta$. The wide allowed range for $\delta$ resulting from a previous experiment [26], is now considerably narrowed and can lead to much more accurate values for the B(E2) $\downarrow$ reduced transition probabilities. Furthermore unambiguous spin assignments have been made for most of the negative parity states up to $2107 \mathrm{keV}$.

In this section, some observed states of ${ }^{45} \mathrm{Sc}$ will be discussed individually. Since no new information was obtained for the $3 / 2^{-}, 376.8 \mathrm{keV}$, the $11 / 2^{-}$, $1237.5 \mathrm{keV}$ and the $15 / 2^{-}, 2106.8 \mathrm{keV}$ levels, except lifetimes (or limits), they will not be discussed individually here. The present angular correlation and lifetime results confirm the spin and parity assignments made in reference [22] for the first level, in references $[26,27]$ for the second level and in reference [27] for the latter one. 
$3.1720 .6 \mathrm{keV}$ LEVEL. - This level decays by a $96.5 \%$ branch to the $7 / 2^{-}$ground state and a $3.5 \%$ branch not reported previously to the $3 / 2^{+}, 12.4 \mathrm{keV}$ state. This level has been seen in the $\left({ }^{3} \mathrm{He}, \mathrm{d}\right)$ reaction [22] where the deuteron angular distribution was fitted with $l_{\mathrm{p}}=3$ implying $I^{n}=5 / 2^{-}, 7 / 2^{-}$. The angular correlation of the $721 \mathrm{keV}$ transition leads to a definite $I^{\pi}=5 / 2^{-}$assignment (Fig. 2) in agreement with Eastham and Phillips [10]. In order to reproduce acceptable E2 transition strengths, $\delta$ values $>15$ and $<-60$ were rejected and the value given in table III adopted. Taking into account the weighted average value $B(E 2) \uparrow=67 \pm 10 \mathrm{e}^{2} \mathrm{fm}^{4}$ deduced from Coulomb excitation experiments [3-10] and the branching ratio and lifetime values, we calculate an absolute value for the mixing ratio $|\delta|=0.084 \pm 0.007$ in excellent agreement with the present one.

$3.21068 .6 \mathrm{keV}$ LEVEL. - In both stripping and pick up reactions $[22,23]$ this state is populated by an $l_{\mathrm{p}}=1$ transition, allowing $I^{\pi}=1 / 2^{-}$and $3 / 2^{-}$. The level decays by a $76 \%$ branch to the $3 / 2^{-}, 376.8 \mathrm{keV}$ level and a $24 \%$ branch to the $5 / 2^{-}, 720.6 \mathrm{keV}$ level ; this last branch was not reported by Zuk et al. [26] who could not resolve the $1068.6 \rightarrow 720.6 \mathrm{keV}$ and $376.8 \rightarrow 12.4 \mathrm{keV}$ transitions due to the use of an $\mathrm{NaI}$ detector. In the present case, the simultaneous analysis of the two reported transitions rules out $I=1 / 2$ and yields a definite $I^{\pi}=3 / 2^{-}$to the level. This confirms the proposed $I=3 / 2$ value of Rust et al. [21] based on the analysis of the ${ }^{45} \mathrm{Sc}\left(\mathrm{p}, \mathrm{p}^{\prime}\right)$ reaction in terms of Hauser-Feshbach theory.

$3.31409 .5 \mathrm{keV}$ LEVEL. - This level was found to decay by a $87 \%$ branch to the $7 / 2^{-}$ground state and by a $13 \%$ branch to the $5 / 2^{-}, 720.6 \mathrm{keV}$ level, in agreement with the results of Blasi et al. [9] using the ${ }^{45} \mathrm{Sc}\left(\mathrm{p}, \mathrm{p}^{\prime} \gamma\right)$ reaction. The last branch was not observed in other experiments. A very weak branch to the $3 / 2^{-}, 376.8 \mathrm{keV}$ level has been reported by Chasman et al. [18] and Rust et al. [21]. This weak transition could not be observed in the present experiment.

The negative parity for this level results from the assigned $L_{\mathrm{np}}=0+2$ orbital angular momentum to the transferred neutron-proton pair in the ${ }^{43} \mathrm{Ca}\left({ }^{3} \mathrm{He}, \mathrm{p}\right)$ reaction [24]. The angular correlation for the $1409.5 \mathrm{keV}$ transition can be fitted at the $0.1 \%$ confidence level by $I=5 / 2,7 / 2$ and $9 / 2$. The present data alone are not sufficient to distinguish between the different spin sequences. The assignment $9 / 2^{-}$ has been proposed by Blasi et al. [9]. In that case, the mixing ratio for the ground state transition would be $\delta=-0.44 \pm 0.07$. However, taking into account the lifetime value given in table II and considering the weak branch to the $3 / 2^{-}, 376.8 \mathrm{keV}$ level, the $9 / 2^{-}$ possibility can be ruled out on the basis of unrealistic M3 enhancement. Moreover, Rust et al. [21] analys- ing the experimental proton angular distribution in terms of the Hauser-Feshbach theory, assigned a probable $7 / 2$ spin to this level.

$3.41557 .1 \mathrm{keV}$ LEVEL. - Only a branch to the $3 / 2^{-}, 1068.6 \mathrm{keV}$ state has been observed in the present work. In view of the limits set on other possible branches, it is in disagreement with the results of Chasman et al. [18] but does not contradict the $\gamma$ decay proposed by Schulte et al. [14] for this level.

This state is populated by an $l_{\mathrm{p}}=1$ transition in both stripping and pick up reactions $[22,23]$ which implies a spin assignment of $1 / 2^{-}$or $3 / 2^{-}$. In the present experiment the angular correlations for the $489 \mathrm{keV}$ transition is consistent with isotropy and thus with a $1 / 2$ assignment but can also be fitted supposing a spin $3 / 2$ for the initial state. Our data alone are not sufficient to give a definite spin assignment. However, according to Rust et al. [21], the experimental cross section for this level in the ${ }^{45} \mathrm{Sc}\left(\mathrm{p}, \mathrm{p}^{\prime}\right)$ reaction gives clear evidence that the spin of the states is $1 / 2$.

$3.51662 .0 \mathrm{keV}$ LEVEL. - This level appears to decay $79 \%$ to the $7 / 2^{-}$ground state and $21 \%$ to the $11 / 2^{-}, 1237.5 \mathrm{keV}$ level. The latter transition has not been reported by Sawa et al. [27] using the same reaction but at higher bombarding energies. The analysis of the $\gamma$-ray angular correlation for the $1662.0 \mathrm{keV}$ transition leads to $I=5 / 2$ or $9 / 2$. Realistic transition strengths for the present observed $1662.0 \rightarrow 1237.5 \mathrm{keV}$ transition are obtained only for a spin $9 / 2$ assignment. In addition, positive parity can be rejected on the basis of unrealistic enhancement for an M2 component in the $1662.0 \mathrm{keV}$ transition. As a result, there is only one solution left : $I^{\pi}=9 / 2^{-}$.

It should be pointed out that the present spinparity assignment as well as the $\delta$ value for the $1662.0 \mathrm{keV}$ transition are in agreement with the quoted values of Sawa et al. [27] based on angular distributions and excitation functions.

3.6 $2092.3 \mathrm{keV}$ LEVEL. - This level was observed in the ${ }^{43} \mathrm{Ca}\left({ }^{3} \mathrm{He}\right.$, p) reaction with an $L_{\mathrm{np}}=0+2$ orbital angular momentum transfer [24]. It implies negative parity for the state. The present angular correlation measurement for the ground state transition can be fitted at the $0.1 \%$ confidence level by $I=7 / 2$ with $\delta=0.51 \pm 0.10$, or $I=5 / 2$ with $\delta=0.05 \pm 0.06$ and $\delta=5.7_{-1.4}^{+2.4}$, or $I=3 / 2$ with $\delta=-0.19 \pm 0.12$. The angular correlation for the $2092.3 \rightarrow 1068.6 \mathrm{keV}$ $\left(3 / 2^{-}\right)$transition yields the following solutions : $I=7 / 2$ with $\delta=0.21_{-0.17}^{+0.23}$, or $I=5 / 2$ with $\delta=0.05 \pm 0.20$ and $\delta=2.3_{-1.0}^{+3.0}$, or $I=3 / 2$ with $\delta<0.24$. On the basis of the lifetime results presented in table II, the $I^{\pi}=3 / 2^{-}$and $I^{\pi}=7 / 2^{-}$possibilities can be rejected because they would imply an enhanced M3 component in the first and second transition respectively. 
Since the positive parity has already been excluded, we are left with an unique $5 / 2^{-}$assignment for the spin and parity of this state; the larger $\delta$ values are not given in table III because they lead to E2 enhancements greater than $100 \mathrm{~W} . \mathrm{u}$.

4. Discussion. - Natural parity states for the ${ }^{45} \mathrm{Sc}$ nucleus have been calculated by McCullen, Bayman and Zamick [1] in the framework of the spherical shell model, considering 5 particles coupled in the $1 f_{7 / 2}$ shell. The effective matrix elements of the residual interaction were estimated on the basis of the ${ }^{42} \mathrm{Sc}$ level scheme as it was known at that time. New calculations have been performed [29], [40] with improved values for the residual interactions. A rough agreement has been found between the energies of the lowest levels with $I \geqslant 7 / 2$ and the calculated values. The measured excitation energies for the $I=3 / 2,5 / 2,1 / 2$ levels are definitely smaller than the calculated values and they appear difficult to reconcile with a pure $\left(1 \mathrm{f}_{7 / 2}\right)^{5}$ configuration for any reasonable set of matrix elements for the residual interaction [29].

This situation is not surprising since the $3 / 2^{-}$ states at 377 and $1069 \mathrm{keV}$ as well as the $1 / 2^{-}$state at $1557 \mathrm{keV}$ contain p-shell admixture in their wave functions as is shown in stripping reactions [22]. An extension of the shell model basis, to include the $p_{3 / 2}, f_{5 / 2}$ and $p_{1 / 2}$ subshells has been attempted recently by McGrory [41] but the calculations were limited to nuclei with $A \leqslant 44$. Figure 4 shows the experimentally established negative parity levels up to about $2.2 \mathrm{MeV}$ of ${ }^{43} \mathrm{Sc},{ }^{45} \mathrm{Sc}$ and ${ }^{47} \mathrm{Sc}$. The data on ${ }^{43} \mathrm{Sc}$ were obtained from reference [42], those on ${ }^{45} \mathrm{Sc}$ from the present work and those on ${ }^{47} \mathrm{Sc}$ from reference [43]. On the left side of the figure are the results of the shell model calculations [41] for $(\mathrm{fp})_{T=1 / 2}^{3}$ states. An improvement in the calculation of the excitation energies is observed as compared with the pure $\left(\mathrm{lf}_{7 / 2}\right)^{3}$ model. However, the complete low-lying odd-parity spectrum cannot be understood.

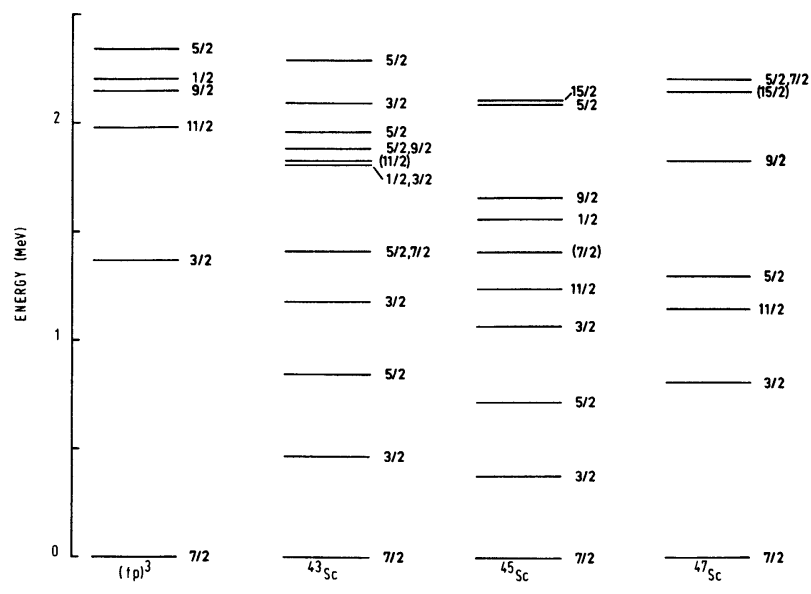

FIG. 4. - Low-lying negative parity levels of the nuclei ${ }^{43} \mathrm{Sc}$

${ }^{45} \mathrm{Sc}$ and ${ }^{47} \mathrm{Sc}$ as presently known. On the left side of the figure are the results of shell-model calculations [42] for ( $\mathrm{fp})^{3}$ states.
In the case of the odd scandium isotopes, the $3 / 2^{+}$ states observed at very low excitation energy, strongly populated by $l_{\mathrm{p}}=2$ transitions in pick up reactions [44], are interpreted as proton holes in the $d_{3 / 2}$ shell. ${ }^{43} \mathrm{Sc},{ }^{45} \mathrm{Sc}$ and ${ }^{47} \mathrm{Sc}$ display $3 / 2^{+}$levels at 151 , 12 and $767 \mathrm{keV}$ respectively. Such low-lying positive parity states suggest that configurations involving particle excitations of the ${ }^{40} \mathrm{Ca}$ core can be rather important for negative parity states as well. In the model of Johnstone [45] and Johnstone and Payne [46], the negative parity states of ${ }^{43} \mathrm{Sc}$ are assumed to arise from the mixture of pure (fp) ${ }^{3}$ and $5 \mathrm{p}-2 \mathrm{~h}$ (five particletwo hole) $K^{\pi}=3 / 2^{-}$rotational band states. An indication of the mixing of the two types of configurations is provided by the results of both the charge exchange reaction ${ }^{43} \mathrm{Ca}\left({ }^{3} \mathrm{He}, \mathrm{t}\right){ }^{43} \mathrm{Sc}$ [47] and $\gamma$-ray spectroscopy in ${ }^{43} \mathrm{Sc}$ [48]. This model can account for the observed normal-parity level scheme. Although similar calculations, involving (fp) ${ }^{5}$ and $7 \mathrm{p}-2 \mathrm{~h}$ states have not yet been attempted for ${ }^{45} \mathrm{Sc}$, we may compare the experimental and theoretical results of ${ }^{43} \mathrm{Sc}$ with the present results of ${ }^{45} \mathrm{Sc}$. The strong similarity between the level schemes of these two nuclei suggest a $K^{\pi}=3 / 2^{-}$band in ${ }^{45} \mathrm{Sc}$ which comprise the levels at $377 \mathrm{keV}\left(3 / 2^{-}\right), 721 \mathrm{keV}\left(5 / 2^{-}\right), 1409 \mathrm{keV}\left(7 / 2^{-}\right)$ and probably the level at $1662 \mathrm{keV}\left(9 / 2^{-}\right)$in addition to states arising chiefly from the (fp) ${ }^{5}$ configuration. On the other hand the level scheme of ${ }^{47} \mathrm{Sc}$ differs appreciably from those of ${ }^{43} \mathrm{Sc}$ and ${ }^{45} \mathrm{Sc}$. However, in that case the energy separation of the first $3 / 2^{+}$core excited state and the ground state is larger than in the other two isotopes. Furthermore the agreement between the sequence of low-lying negative parity levels and the $\left(1 \mathrm{f}_{7 / 2}\right)$ calculations performed by Brut [41] is quite good. This suggests that the contribution of the two hole states is less important than in ${ }^{43} \mathrm{Sc}$ and ${ }^{45} \mathrm{Sc}$ due to the neutron excess.

Another approach to the theoretical understanding of ${ }^{45} \mathrm{Sc}$ is provided by the strong coupling model of Bohr and Mottelson [49] which has been reasonably successfully applied to various nuclei in the $1 f_{7 / 2}$ shell in spite of its great simplicity [2], [50, 51]. The calculations of Malik and Scholz [2] for the ${ }^{45} \mathrm{Sc}$ nucleus show a better agreement with the experimental data than the simple $\left(1 f_{7 / 2}\right)^{5}$ shell model. Unfortunately no transition probabilities have been calculated, therefore a detailed comparison with experiment is not possible. Calculations performed in the present study are based on the same model. The Nilsson Hamiltonian was used to describe the single particle motion. The lowest intrinsic state in ${ }^{45} \mathrm{Sc}$ is described with four neutrons coupled to $K=0$ in the Nilsson orbitals No. $14(K=1 / 2)$ and No. 13 $(K=3 / 2)$ and the odd proton in the orbital No. 14 $(K=1 / 2)$. This unpaired proton was allowed to occupy any orbit in the $N=3$ oscillator shell. The ground state band has therefore $K=1 / 2$ but, due to the Coriolis coupling among different bands, the spin of the ground state differs from $1 / 2$. Details on the 
model as well as explicit relations for the matrix elements are given in reference [52]. The parameters $C$ and $D$ used in the Nilsson Hamiltonian are equal to -1.77 and -0.133 [51] consistant with the single particle energies as deduced from ${ }^{41} \mathrm{Sc}$ and ${ }^{49} \mathrm{Sc}$. The value of the deformation parameter $\delta=0.14$, close to the minimum of the total ground state energy, and the rotational constant $\hbar^{2} / 2 J=90 \mathrm{keV}$ give the best fit between the calculated and observed spectrum. In figure 5 the experimental negative parity level scheme is compared to the calculated one. All levels are given in the theoretical spectrum up to $2.2 \mathrm{MeV}$ and only states with $I>13 / 2$ are reported at higher excitation energy. Electromagnetic properties were also calculated and the results of the calculations are given along with the experimental values in table IV. The free nucleon charge and gyromagnetic ratios were used, while a value of $Z / A$ was adopted for the rotational gyromagnetic ratio. Values of $-0.21 \mathrm{~b}$ and 5.36 n.m. are obtained for the electric quadrupole and magnetic dipole moments of the ground state which have to be compared to the experimental values [53] of $-0.22 \pm 0.01 \mathrm{~b}$ and $4.75633 \pm 0.00001$ n.m.

A survey of both figure 5 and table IV leads to two statements. First of all, a definite improvement is obtained for the energy spectrum : up to $2.2 \mathrm{MeV}$ excitation energy, the model accounts for all the negative parity levels. On the other hand, several calculated transition probabilities disagree with the experimental data. At this point, it should be reminded that for several other nuclei of the $1 f_{7 / 2}$ shell, the present model successfully reproduced the electromagnetic properties for states having a predominant ground state configuration [51]. This fact appears also in the present investigation where the main contribution to the wave functions of the first $7 / 2^{-}, 3 / 2^{-}$, $11 / 2^{-}$and $1 / 2^{-}$states corresponds to the unperturbed

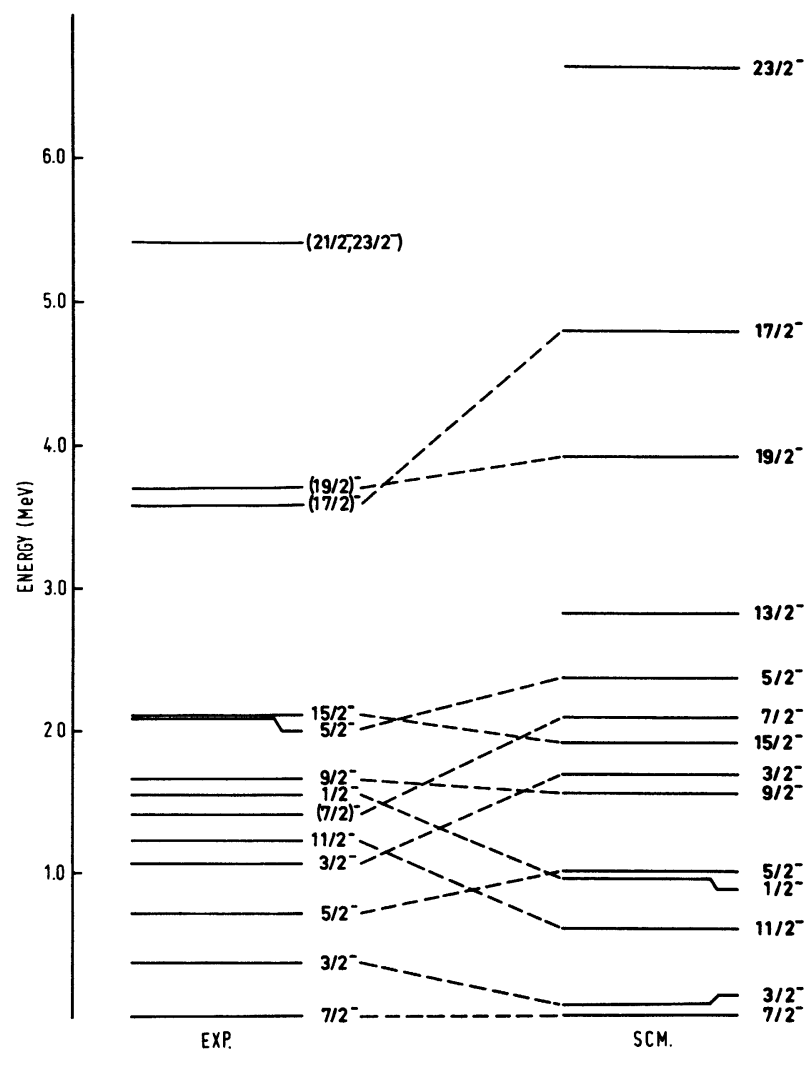

FIG. 5. - Comparison of the calculated negative parity energy levels in the framework of the strong coupling model (SCM) with experiment. The levels of angular momentum $I>15 / 2$ are from reference [29].

$K^{\pi}=1 / 2^{-}$ground state band. The difficulties encountered for reproducing transition probabilities for other levels may arise from the omission of other types of excitation. Thus, the model explicitly treats the excitations of the unpaired proton from the Nilsson orbital No. 14 to all the other orbitals of the (fp) shell

TABLE IV

$B(\mathrm{M} 1)$ and $B(\mathrm{E} 2)$ reduced matrix elements for transitions between negative parity states in ${ }^{45} \mathrm{Sc}$

\begin{tabular}{|c|c|c|c|c|c|c|c|}
\hline \multirow{2}{*}{$\begin{array}{c}E_{\mathrm{i}} \\
(\mathrm{keV})\end{array}$} & \multirow{2}{*}{$\begin{array}{c}E_{\mathrm{f}} \\
(\mathrm{keV})\end{array}$} & \multirow[t]{2}{*}{$I_{\mathrm{i}}^{\pi}$} & \multirow[t]{2}{*}{$I_{\mathrm{f}}^{\pi}$} & \multicolumn{2}{|c|}{$B(\mathrm{M} 1)\left(\mu_{\mathrm{N}}^{2}\right)$} & \multicolumn{2}{|c|}{$B(\mathrm{E} 2)\left(\mathrm{e}^{2} \mathrm{fm}^{4}\right)$} \\
\hline & & & & Exp. & Th. & Exp. & Th. \\
\hline- & - & - & - & - & & - & \\
\hline 377 & 0 & $3 / 2^{-}$ & $7 / 2^{-}$ & & & $144 \pm 8$ & 146 \\
\hline 721 & 0 & $5 / 2^{-}$ & $7 / 2^{-}$ & $0.45 \pm 0.05$ & 0.87 & $89 \pm 14$ & 10 \\
\hline 1069 & 377 & $3 / 2^{-}$ & $3 / 2^{-}$ & $0.26 \pm 0.09$ & 0.25 & $93_{-73}^{+171}$ & 10 \\
\hline & 721 & & $5 / 2^{-}$ & $0.65_{-0.23}^{+0.27}$ & 0.45 & $<3074$ & 55 \\
\hline 1237 & 0 & $11 / 2^{-}$ & $7 / 2^{-}$ & & & $104 \pm 8$ & 81 \\
\hline 1409 & 0 & $7 / 2^{-}$ & $7 / 2^{-}$ & $0.04 \pm 0.01$ & 0.13 & $>\overline{12}$ & 0.32 \\
\hline & 721 & & $5 / 2^{-}$ & $<0.082$ & 0.60 & $<2487$ & 24 \\
\hline 1557 & 1069 & $1 / 2^{-}$ & $3 / 2^{-}$ & $<1.69$ & 3.4 & $<10^{5}$ & 21 \\
\hline 1662 & 0 & $9 / 2^{-}$ & $7 / 2^{-}$ & $0.05 \pm 0.01$ & 0.24 & $60_{-16}^{+20}$ & 15 \\
\hline 2092 & 0 & $5 / 2^{-}$ & $7 / 2^{-}$ & $0.64_{-9}^{+112}$ & 0.05 & $<30$ & 1.3 \\
\hline & 377 & & $3 / 2^{-}$ & $<0.145$ & 0.04 & $<708$ & 1.5 \\
\hline & 1069 & & $3 / 2^{-}$ & $0.66 \pm 0.25$ & 0.87 & $<735$ & 14 \\
\hline 2107 & 1237 & $15 / 2^{-}$ & $11 / 2^{-}$ & & & $<206$ & 81 \\
\hline
\end{tabular}


but not the core excitations. Such excitations should be implicitly taken into account in a phenomenological way and this may explain the fair agreement obtained for the energy spectrum. However, the transition probabilities are much more sensitive to the details of the wave function. A better agreement for the electromagnetic properties may be obtained taking into account explicitly the $7 \mathrm{p}-2 \mathrm{~h}$ component.

Note added in proof :

A value of $(30 \pm 3)$ ps has been measured for the mean life the $2107 \mathrm{keV}$ level by Bini, Blasi and Kutschera (private communication), resulting in a $B(E 2) \downarrow$ value of $55 \pm 5 \mathrm{e}^{2} \mathrm{fm}^{4}$.

\section{References}

[1] McCullen, J. D., Bayman, B. F. and Zamick, L., Phys. Rev. B 134 (1964) 51

[2] Malik, F. B. and Scholz, W., Phys. Rev. 150 (1966) 919.

[3] Goldberg, M. D. and Hooton, B. W., Nucl. Phys. A 132 (1969) 369

[4] Afonin, O. F., Grinberg, A. P., Lemberg, I. K. and ChuGUNOv, I. N., Sov. J. Nucl. 6 (1968) 160.

[5] Alkhazov, D. G., Grinberg, A. P., GusenskiI, G., Erokhina, E. and Lemberg, I. K., Sov. Phys. JETP 37 (1960) 1086.

[6] Blaugrund, A. E., Holland, R. E. and Lynch, F. J., Phys. Rev. 159 (1967) 926.

[7] Imanishi, N., Fukazawa, F., Sakisaka, M. and Uemura, Y., Nucl. Phys. A 101 (1967) 654

[8] Andreev, D. S., Afonin, O. F., Bondarev, V. K., GrinBerg, A. P., Erokhina, K. I. and Lemberg, I. K., Bull. Acad. Sci. USSR Phys. Ser. 32 (1969) 1543.

[9] Blasi, P., Fazzini, T. F., Maurenzig, P. R., Taccetti, N. and RICCI, R. A., Nuovo Cimento 68A (1970) 49.

[10] Eastham, D. A. and Phillips, W. R., Nucl. Phys. A 146 (1970) 112.

[11] GFöller, D. and Flammersfeld, A., Z. Phys. 187 (1965) 490.

[12] Porter, F. T., Freedman, M. S., Wagner, F. and OrlanDINI, K. A., Phys. Rev. 146 (1966) 774.

[13] ERLandsson, B., Ark. Fys. 34 (1967) 293.

[14] Schulte, R. L., King, J. D. and Taylor, H. W., Can. J. Phys. 52 (1974) 131.

[15] Schulte, R. L., King, J. D. and Taylor, H. W., Phys. Rev. C 9 (1974) 1436.

[16] Exström, P., Erlandsson, B., Marcinkowski, A. and Tillmann, J., Z. Phys. 252 (1972) 189.

[17] Mani, G. S., Nucl. Phys. A 177 (1971) 197.

[18] Chasman, C., Jones, K. W. and Ristinen, R. A., Phys. Rev. 173 (1968) 1072.

[19] Broman, L., Al-Jadir, M. N. I., Larsson, C. and Ott, A., Ark. Fys. 40 (1968) 223.

[20] ZuK, W. M., Davidson, W. F., Najam, M. R. and Awal, M. A., Z. Phys. 242 (1971) 93.

[21] Rust, N. J. A., NAudé, W. J., Koen, J. W. and Mouton, W. L., Nucl. Phys. A 219 (1974) 232.

[22] Schwartz, J. J. and Alford, W. P., Phys. Rev. 149 (1966) 820.

[23] Ohnuma, H., Phys. Rev. C 3 (1971) 1192.

[24] Hardie, G., Gloeckner, D., MeYer-Schützmeister, L. and Braid, T. H., Phys. Rev. C 10 (1974) 1829.

[25] Mairle, G. and Wagner, G. J., Z. Phys. 251 (1972) 404.

[26] Zuk, W. M., Symons, G. D. and Baugh, D. J., Aust. J. Phys. 24 (1971) 13.

[27] Sawa, Z. P., Blomevist, J. and Gullholmer, W., Nucl. Phys. A 205 (1973) 25.
[28] Metzger, F. R., Phys, Rev. C 12 (1975) 312

[29] Bizzeti, P. G., Bizzeti-Sona, A. M., Bucciolini, M., Huber, R., Kutschera, W., Morinaga, H., RicCi, R. A. and Signorini, C., Nuovo Cimento 26A (1975) 25.

[30] Haas, B., Chevallier, J., Britz, J. and Styczen, J., Phys. Rev. C 11 (1975) 1179.

[31] Ball, G. C., Forster, J. S., Ingebretsen, F. and Monahan, C. F., Can. J. Phys. 48 (1970) 2735.

[32] Poletti, A. R. and Warburton, E. K., Phys. Rev. B 137 (1965) 595.

[33] Rose, H. J. and Brink, D. M., Rev. Mod. Phys. 39 (1967) 306.

[34] Endt, P. M. and van der Leun, C., Nucl. Phys. A 235 (1974) 27.

[35] Lindhard, J., ScharfF, M. and SchitT, H. E., K. Danske Vidensk. Selsk. Mat.-Fys. (Medd. and Skr.) 33 (1963) No. 14.

[36] Blaugrund, A. E., Nucl. Phys. 88 (1966) 501.

[37] Broude, C., Beck, F. A. and Engelstein, P. E., Nucl. Phys. A 216 (1973) 603

[38] Haas, F., Freeman, R. M., Heusch, B. and Gallmann, A., Bull. Am. Phys. Soc. 20 (1975) 693.

[39] Ball, G. C., Forster, J. S., Ward, D. and Monahan, C. F., Phys. Lett. B 37 (1971) 366.

[40] Brut, F., Thesis, Grenoble (1972).

[41] McGrory, J. B., Phys. Rev. C 8 (1973) 693

[42] Endt, P. M. and Van Der Leun, C., Nucl. Phys. A 214 (1973) 1.

[43] Toulemonde, M., Deschênes, L., Jamshidi, A. and Schulz, N., Nucl. Phys. A 227 (1974) 309.

[44] LewIs, M. B., Nucl. Data B 4 (1970) 237.

[45] Johnstone, I. P., Nucl. Phys. A 110 (1968) 429.

[46] Johnstone, I. P. and Payne, G. L., Nucl. Phys. A 124 (1969) 217.

[47] Alford, W. P., Schulz, N. and Jamshidi, A., Nucl. Phys. A 174 (1971) 148

[48] Manthuruthil, J. C., Poirier, C. P. and Walinga, J., Phys. Rev. C 1 (1970) 507.

[49] Bohr, A. and Mottelson, B. R., K. Danske Vidensk. Selsk. Mat.-Fys. (Medd. and Skr.) 27 (1953) No. 16.

[50] Zürmuhle, R. W., Hutcheon, D. A. and Weaver, J. J., Nucl. Phys. A 180 (1972) 417.

[51] HaAs, B., Taras, P. and StYCZen, J., Nucl. Phys. A 246 (1975) 141

[52] Haas, B. and Taras, P., Can. J. Phys. 52 (1974) 49.

[53] ShIRLEY, V. S., Hyperfine Interactions in Excited Nuclei, edited by Goldring G. and Kalish R. (Gordon and Breach, New York) 1971 , p. 1261 